\title{
Unidentified infrared bands and the formation of PAHs around carbon stars
}

\author{
Angela Speck $^{1}$, Mike Barlow ${ }^{2}$, Roger Wesson ${ }^{2}$, Geoff Clayton ${ }^{3}$, and \\ Kevin Volk ${ }^{4}$ \\ ${ }^{1}$ Physics \& Astronomy Department, University of Missouri- Columbia \\ email: speckan@missouri.edu \\ ${ }^{2}$ Physics \& Astronomy Department, University College London \\ ${ }^{3}$ Dept. of Physics \& Astronomy, Louisiana State University \\ ${ }^{4}$ Gemini Observatory
}

\begin{abstract}
Although unidentified infrared bands (UIBs) have been observed in many astrophysical environments, there is one notable exception: carbon (C) stars. Only a handful of $\mathrm{C}$ stars have been shown to emit UIBs and most have hot companions. This makes $\mathrm{C}$ stars with hot companions an ideal location to investigate the emitters of the UIBs. PAHs are excited by absorption of single photons whose energy is then distributed over the whole molecule. These molecules then emit the energy at the characteristic wavelengths, but the precise wavelengths and strength ratios depend on the size, composition and charge state of the individual PAHs. Furthermore, the wavelength of photons needed to excite PAHs depends on their size and charge state. While small PAHs undoubtedly need higher energy (UV) photons, it has been suggested that large or ionized PAHS ( $>100 \mathrm{C}$ atoms) can be excited by visible or even near-IR photons. The lack of PAH emission from single carbon stars suggests that either PAHs do not form around $\mathrm{C}$ stars or that only small neutral grains form, which cannot be excited by a $\mathrm{C}$ star's radiation field.

There are two competing formation mechanisms for PAHs around C stars: (1) "bottom-up" where acetylene molecules react to form aromatic rings, building up to PAHs; or (2) "top-down", where small carbon grains react with $\mathrm{H}$ atoms and desorb PAHs

Using spatially resolved spectroscopic observations from Gemini/Michelle, of five carbon stars with hot companions, we investigate the circumstance under which PAH emission occurs and try to discriminate between formation mechanisms.
\end{abstract}

\section{Discussion}

ZINNER: Average carbon-rich AGB stars produce silicon carbide, not graphite. If we take a look at isotropic distribution you, find in silicon carbide grains in meteorites have ${ }^{12} \mathrm{C}$ to ${ }^{13} \mathrm{C}$ ratios of between 40 and 80 , and this agrees with what you see in average carbon stars. But the isotropic composition of the meteoritic graphite grains which are from AGB stars have much higher ${ }^{12} \mathrm{C}$ to ${ }^{13} \mathrm{C}$ ratios, up to 300,500 or so. The reason is that together with the high ${ }^{12} \mathrm{C}$ to ${ }^{13} \mathrm{C}$ ratio you also have to have high $\mathrm{C} / \mathrm{O}$ ratio. So I think these graphite grains are preferentially formed from AGB stars with a very high $\mathrm{C} / \mathrm{O}$ ratio.

SPECK: I think this fits in with what I was showing about extreme carbon stars. Regardless of the $\mathrm{C} / \mathrm{O}$ ratio, because of the high pressure and the mass loss rates, you can form graphite easily there. Since extreme carbon stars are optically obscured, the measurement of $\mathrm{C} / \mathrm{O}$ ratio is hard. 
AlaxANDER: In order to form benzene and PAHs in circumstellar envelopes by acetylene addition, you have to make them under a narrow temperature range about 900 to $1000 \mathrm{~K}$ and it takes $10^{6}$ years which is much too long for AGB evolution.

SPECK: Something that I am slightly disturbed about is the issue of the temperature. It does seem that there is a peak in that zone around $1000 \mathrm{~K}$ which suggests that the reactions are happening in stars of low mass loss rates. I think that the work Isabelle did 15 years ago may be under-estimating the formation rate because some of the conditions they chose were not really appropriate to carbon stars.

ZIURYS: You see these UIR PAH bands in young planetary nebula, but do you see them in old planetary nebulae, like the Helix?

SpECK: The problem with the Helix is that you're running into other issues such as clumping. I don't know the answer to the question in terms of exactly at what age the UIR bands start to diminish.

HenNing: In the paper by Goto, she finds that in carbon-rich post-AGB stars, the extent of the aliphatic zone is decreasing as you go to later stages of evolution. So spatial information is available through adaptive optics observations, at least for this phase of stellar evolution.

SPECK: I think by looking at the range of stars where they have a similar radiation field but naturally different separations, we can really start to get a feeling for the conditions on how these things form and evolve.

HENNING: Is there any evidence with 3.4 micron absorption feature in some of the carbon stars or AGB stars? At some point you would expect to see at least some of the objects with absorption features.

SPECK: I would say 'no' even in the extreme carbon stars where we have got ISO spectra and should be able to detect any absorption feature. I don't think we see anything that suggests aliphatic stuff. We see acetylene and HCN, that's it. 